\title{
Early temperament as a predictor of language skills at 40 months
}

\author{
Yuuka Ishikawa-Omori ${ }^{1}$, Tomoko Nishimura ${ }^{1,2}$, Atsuko Nakagawa ${ }^{3}$, Akemi Okumura ${ }^{1,2}$, Taeko Harada 1,2, \\ Chikako Nakayasu' ${ }^{2}$, Toshiki Iwabuchi 1,2, Yuko Amma², Haruka Suzuki², Mohammad Shafiur Rahman 1,2, \\ Ryuji Nakahara², Nagahide Takahashi, ${ }^{1,2}$, Yoko Nomura ${ }^{2,4,5}$ and Kenji J. Tsuchiya ${ }^{1,2^{*}}$
}

\begin{abstract}
Background: Mastering language involves the development of expressive and receptive skills among children. While it has been speculated that early temperament plays a role in the acquisition of language, the actual mechanism has not yet been explored. We investigated whether temperament at 18 months predicted expressive or receptive language skills at 40 months.

Methods: A representative sample of 901 children and their mothers who were enrolled and followed-up longitudinally in the Hamamatsu Birth Cohort for Mothers and Children study was included in the analysis. Child temperament was measured at 18 months using the Japanese version of the Early Childhood Behavior Questionnaire. Expressive and receptive language skills were measured at 40 months using the Mullen Scales of Early Learning.

Results: The multiple regression analysis, adjusting for potential confounders, suggested that higher motor activation (fidgeting) at 18 months was associated with lower expressive and receptive language skills at 40 months. Higher perceptual sensitivity was associated with higher expressive and receptive language skills at 40 months.

Conclusions: Specific temperament at 18 months of age predicted the development of the child's expressive and receptive language skills at 40 months.
\end{abstract}

Keywords: Language skills, Temperament, Cohort study, Early childhood behavior questionnaire (ECBQ)

\section{Background}

Language plays a key role in understanding child neurodevelopment $[1,2]$. Studies have suggested that language acquisition progresses significantly during the first 4 years of life [3], while language skills in early childhood are known to predict cognitive skills at school age [4] and academic performance in young adulthood [5]. Language skills in early childhood are influenced by individual characteristics $[6,7]$ of both the child and environment, including the family; for example, the child's sex [8], birth

${ }^{*}$ Correspondence: tsuchiya@hama-med.ac.jp

${ }^{2}$ Research Center for Child Mental Development, Hamamatsu University School of Medicine, Handayama 1 Higashiku, Hamamatsu 4313192, Japan Full list of author information is available at the end of the article weight [9], gestational age at birth [10], birth order [11], maternal age [12], maternal education [13], maternal history of mood or anxiety disorders $[14,15]$, and household income $[8,10]$. Child temperament is also a candidate factor that can influence child language development [16-18].

In the literature, temperament has been understood as "the constellation of inborn traits that determine a child's unique behavioral style" [19]. Along with this understanding, temperament has been measured in a few different ways. For instance, Goldsmith attempted to define it based on the emotionality of a child aged 16 to 36 months [20], although emotionality is not the only trait that determines an individual style of behavior; in particular, this is not the case for young children. 
In a hallmark study of temperament of young children, including those in the first year of life, Rothbart expanded the original concept and re-defined child temperament as individual differences in emotional, motor, and attentional reactivity and self-regulation [21]. Subsequent studies have suggested that temperamental characteristics appear as early as infancy and are driven by biological factors such as genes and brain structure [21, 22], out of which personality grows later in life [21]. Child temperament has also been studied as a predictor of physical and mental health problems and adjustment in later childhood and adolescence since it is observed early in development [21]. Among the many temperament scales developed [23], those developed by Rothbart and her colleagues, namely the Infant Behavior Questionnaire [24] for infants between 3 and 12 months of age and the Early Childhood Behavior Questionnaire (ECBQ; [25]) for children between 18 months and 3 years of age, are the most widely used parent-report measures assessing early childhood temperament [24]; these scales measure 21 fine-grained temperament dimensions [26]. Until recently, early child temperament has been reported to reflect early attentional skills, which is the basis for selfregulation $[21,27]$. To this end, studies have shown that child language skills are associated with early attentional skills and self-regulation [28, 29], supporting the finding that temperament can be a determinant of language skills development [30]. In other words, exploring the association between child temperament and language skills development elucidates the mechanism of child language development.

Despite the growing recognition of child temperament's role in language development in recent years, the link between them remains unclear (e.g., [16-18]). To our knowledge, four longitudinal studies have investigated whether child temperament was associated with language skill development using the temperament scale developed by Rothbart and colleagues [16, 17, 31, 32]. All four studies supported the association, but unclarity remained regarding which subscale of temperament was associated with language skills. Potential reasons behind this inconsistency include 1) the relatively modest sample sizes; for instance, the largest size was 148 in the study by Davison and colleagues [31], followed by 142 [17], 70 [32], and 47 [16]; 2) insufficient control of covariates including sex, birth weight, birth order, maternal age, maternal education, and household income; and 3) language skill was assessed only from the aspect of expressive skills (language productivity). Furthermore, several studies have reported associations between child temperament and expressive and receptive language skills during infancy and early childhood (e.g., [18, 33-36]). Unfortunately, these studies did not adopt scales developed by
Rothbart [18,33] and were cross-sectional [34-36]; thus, longitudinal studies are required to investigate whether the association is causal. To date, few studies have examined whether early temperament is related to language development using a longitudinal design (e.g., [16, 17, $31,32]$ ), and no specific subscales of temperament have been shown to be consistently associated with language development.

Due to the paucity and inconsistency of the findings thus far, we attempted to investigate whether child temperament at 18 months predicts expressive or receptive language skills at 40 months. To this end, we conducted an exploratory study with a relatively large sample size using a longitudinal design to evaluate the link between early temperament and expressive or receptive language skills, corresponding to language productivity and verbal comprehension, respectively. Furthermore, we controlled for covariates including sex, birth weight, birth order, maternal age, maternal education, and household income, which have previously been reported to be associated with language skills. As this study is exploratory, we treat all subscales equally without emphasizing specific subscales of temperament, considering the comparability of our findings to the existing literature.

\section{Methods \\ Participants}

The participants were mothers and their newborn infants, with mothers enrolled in the Hamamatsu Birth Cohort for Mothers and Children (HBC Study; [37, 38]) during pregnancy and infants being enrolled at birth. Both were monitored until the child was approximately 8 years old and continue to be monitored. Based on official national statistics, participants in this cohort are considered a representative sample of the general population of Japan; the enrolled mothers are representative of Japanese mothers with respect to age, socioeconomic status, and parity, and their children are representative of Japanese children with respect to birth weight and gestational age at birth [37, 38].

We contacted all the pregnant women $(N=1138)$ enrolled in the HBC Study who were expected to give birth, either at Hamamatsu University Hospital or Kato Maternity Clinic (both in Hamamatsu City), or who gave birth between December 24, 2007, and March 19, 2012. Nineteen mothers gave multiple births, 100 mothers entered the cohort twice, and one mother entered the cohort three times for a total of 1258 participating children. Moreover, 357 infants were excluded because of their absence at the 18 months examination when child temperament was measured or at the 40 months examination when language skills were measured, resulting in 
901 out of 1258 infants (72\%) being included in the current study.

The study was conducted in accordance with the guidelines of the World Medical Association Declaration of Helsinki and was approved by the Medical Ethical Committee of Hamamatsu University School of Medicine, Japan (No. 20-82, 21-114, 22-29, 24-67, 24-237, 25-143, 25-283, E14-062, 17-037, 17-037-3, 20-233). Written informed consent was obtained from all caregivers (mothers [except for three participating children], two grandmothers, and one father) for their own participation in the study and that of their infants.

\section{Materials and procedures Child temperament}

To measure child temperament, we administered the Japanese version of the ECBQ [25] at the age of 18 months. In a sample of Japanese children, Cronbach's alpha coefficients of the 18 subscales ranged from 0.59 to 0.90 [39]. Both the Japanese version and original ECBQ included a parent-report questionnaire of 201 items and a threefactor structure of negative affectivity, effortful control, and surgency/extraversion [39]. Each of the three factors comprised several subscales, identical to the original ECBQ (Table 1). Our analyses focused on these 18 subscales and not on the three higher-order factors. This is because most of the existing literature used subscales (e.g., [16, 17, 31, 32]). Comparison with previous studies is easier by focusing on subscales than using higher-order factors. Each subscale described one specific aspect of behavior (e.g., "In a situation where s/he is meeting new people, how often did your child become quiet?" or “During everyday activities, how often did your child appear to listen to even very quiet sounds?") and was rated on a 7-point scale ranging from "never" to "always," depending on the frequency of its occurrence. To enhance the objectivity of parental assessments, caregivers were first asked to complete the ECBQ; hereafter, a face-to-face interview was conducted with them by an examiner of our research team.

\section{Expressive and receptive language skills}

The assessment of language development in early childhood requires evaluation of both expressive and receptive language skills [3]. We used two subscales of the Mullen Scales of Early Learning (MSEL; [40]). In the MSEL, expressive language skills are defined as those that support the ability to use language productively, such as speaking, and receptive language skills are defined as those that promote processing of linguistic input, such as

Table 1 Definition of subscales: Early Childhood Behavior Questionnaire (ECBQ)

\begin{tabular}{ll}
\hline Factor/Subscales & Definition \\
\hline $\begin{array}{l}\text { Negative affectivity } \\
\text { Discomfort }\end{array}$ & Amount of negative affect related to sensory qualities of stimulation. \\
Fear & Negative affect related to anticipated pain, distress, sudden events and/or potentially threatening situations. \\
Frustration & Negative affect related to interruption of ongoing tasks or goal blocking. \\
Motor activation & Repetitive small-motor movements; fidgeting. \\
Perceptual sensitivity & Detection of slight, low-intensity stimuli from the external environment. \\
Sadness & Tearfulness or lowered mood related to suffering, disappointment, or loss. \\
Shyness & Slow or inhibited approach and/or discomfort in social situations involving novelty or uncertainty. \\
Soothability & Rate of recovery from peak distress, excitement, or general arousal. \\
Effortful control & \\
Attentional focusing & Sustained duration of orienting on an object of attention; resisting distraction. \\
Attentional shifting & The ability to transfer attentional focus from one activity/task to another. \\
Cuddliness & Child's expression of enjoyment in and molding of the body to being held by a caregiver. \\
Inhibitory control & The capacity to stop, moderate, or refrain from a behavior under instruction. \\
Low-intensity pleasure & Pleasure or enjoyment related to situations involving low intensity, rate, complexity, novelty, and incongruity. \\
Surgency/extraversion & \\
Activity level & Level (rate and intensity) of gross motor activation, including rate and extent of locomotion. \\
High-intensity pleasure & Pleasure or enjoyment related to situations involving high intensity, rate, complexity, novelty, and incongruity. \\
Impulsivity & Speed of response initiation. \\
Positive anticipation & Excitement about expected pleasurable activities. \\
Sociability & Seeking and taking pleasure in interactions with others. \\
\hline
\end{tabular}

Note. Adapted from “Measurement of fine-grained aspects of toddler temperament: The Early Childhood Behavior Questionnaire," by Putnam SP, Gartstein MA, and Rothbart MK, Infant Behavior Development 2006; 29(3), p. 399 (https://doi.org/10.1016/j.infbeh.2006.01.004). Copyright 2006 by Elsevier Inc 
verbal comprehension [40, 41]. We previously developed $\mathrm{z}$-scores for each MSEL subscale in Japanese children using our HBC Study sample, as the US version of the normative data did not correspond well with the Japanese sample [42]. In the current study, we used the standardized version for Japanese children, in which the internal consistency of expressive and receptive language scores at 40 months was alpha $=0.84$, comparable to the original US version. The $\mathrm{z}$-scores had a mean of 0 and a standard deviation of 1 and were derived from the standardized scores prepared in our previous study [42].

\section{Covariates}

Sex [8], birth weight [9], gestational age at birth [10], birth order [11], maternal age at childbirth [12], maternal education [13], household income at birth [8, 10], and maternal history of mood or anxiety disorders [14, 15], previously reported to be associated with language skills in general, were treated as covariates. Information on the demographic and socioeconomic characteristics of the mothers (maternal age, years of maternal education, annual household income, and maternal history of mood or anxiety disorder) was collected through face-toface interviews during the second trimester of the index pregnancy and in the first 1 to 2 months after childbirth. Information on the infants' sex, gestational age, birth weight, and date of birth was obtained directly from medical records.

\section{Analyses}

Multiple regression analyses were performed to examine the associations between each of the 18 subscales of the ECBQ and the expressive and receptive language scales at 40 months. First, univariate regression analyses were performed to test the association of each subscale with expressive and receptive language scores (Model 1). Hereafter, all 18 subscale scores were analyzed simultaneously in a single model (Model 2). Finally, covariates were added to the model (Model 3). We did not remove statistically non-significant covariates from Model 3 because it is recommended that, in studies exploring risk factors, any established risk factors for the outcome should be retained in the model regardless of the statistical significance [43].

Stata version 13.1 was utilized for all analyses. We used $p$-values to analyze the data, as these are meaningful in exploratory analysis [44]. Considering the adjustments for multiple testing, we defined $p$-values $<.0013$ (= $.05 / 36)$ to be statistically significant to allow for measurements of 18 temperamental subscales and both language skills (i.e., expressive and receptive language).

\section{Results}

\section{Participant characteristics}

Table 2 shows the demographic characteristics of the children and their parents included in the analysis. Comparisons of the 901 participants in the final analytic sample and the 357 children excluded from the analyses revealed no significant differences in sex, birth weight, gestational age at birth, distribution of birth order, nor twin births (see Supplementary Table 1). However, when comparing the mothers in the two groups, the following significant differences were found: the mean age of the mothers at the child's birth was significantly lower among excluded children $(M=30.4, S D=5.0)$ than participating children $(\mathrm{M}=31.9, \mathrm{SD}=5.0 ; \mathrm{z}=-4.33, p<.001)$ and annual household income was significantly lower among excluded children $(\mathrm{M}=5.78, \mathrm{SD}=2.84$ million JPY $)$ than participating children $(\mathrm{M}=6.13, \mathrm{SD}=2.82$ million JPY; $\mathrm{z}=-2.36, p=.02)$.

All 18 ECBQ subscales were graded from 1 to 7 , with some variations in the mean values. We found correlations among the 18 subscales (see Supplementary Table 2). The mean $\mathrm{z}$-scores of expressive and receptive language skills at 40 months were all close to 0 , but not exactly 0 . This was expected because, in our analysis, we excluded 357 children from the original sample of the HBC Study, from which the Japanese version of the normative data were derived.

\section{ECBQ subscales and expressive language skills at $\mathbf{4 0 \text { months }}$}

Table 3 shows the regression coefficients and 95\% confidence intervals for each of the subscales in Models 1, 2, and 3 (all the coefficients estimated in Models 1 to 3 are shown in Supplementary Table 3). In Model 1 (univariate), six subscales were significantly associated with expressive language scores. However, in Model 2 (controlling for all 18 subscale scores), only two subscales were significantly associated with expressive language scores, while the remaining subscales were nonsignificant. After controlling for all covariates (Model 3), the motor activation subscale was negatively associated with expressive language, and the perceptual sensitivity subscale was positively associated with expressive language.

\section{ECBQ subscales and receptive language skills at $\mathbf{4 0}$ months}

Table 4 shows the regression coefficients and 95\% confidence intervals for each of the subscales in Models 1, 2, and 3 (all the coefficients estimated in Models 1 to 3 are shown in Supplementary Table 4). In Model 1, four subscales were significantly associated with receptive language scores. In Model 2, two subscales (motor activation and perceptual sensitivity) were significantly associated with receptive language scores, while all other 
Table 2 Demographic characteristics of the children and parents included in the analysis $(N=901)$

\begin{tabular}{|c|c|c|}
\hline & $\mathrm{n}(\%)$ or $\mathrm{M}(\mathrm{SD})$ & Range \\
\hline Child sex (Boys \%) & $450(50 \%)$ & \\
\hline Birthweight (g) & $2931(441)$ & $1064-4286$ \\
\hline Gestational age at birth (weeks) & $38.9(1.6)$ & $29.6-42.1$ \\
\hline \multicolumn{3}{|l|}{ Birth order } \\
\hline First & $447(50 \%)$ & \\
\hline Second & $337(37 \%)$ & \\
\hline Third or Later & $117(13 \%)$ & \\
\hline Twin births & $29(3 \%)$ & \\
\hline Age of mother at the time of the child's birth (years) & $31.9(5.0)$ & $17.7-44.9$ \\
\hline Mother's education (years) & $13.9(1.9)$ & $6.0-23.0$ \\
\hline History of maternal psychiatric diagnosis (Yes \%) & $90(10 \%)$ & \\
\hline Annual household income (million JPY) & $6.13(2.82)$ & $1.0-27.0$ \\
\hline \multicolumn{3}{|l|}{ The ECBQ scores at 18 months of age } \\
\hline Discomfort & $2.0(0.7)$ & $1.0-5.2$ \\
\hline Fear & $2.3(0.7)$ & $1.0-5.1$ \\
\hline Frustration & $3.3(0.9)$ & $1.0-7.0$ \\
\hline Motor activation & $2.2(0.8)$ & $1.0-5.5$ \\
\hline Perceptual sensitivity & $3.3(1.0)$ & $1.0-6.4$ \\
\hline Sadness & $2.5(1.0)$ & $1.0-6.0$ \\
\hline Shyness & $3.5(1.1)$ & $1.0-6.8$ \\
\hline Soothability & $5.7(0.9)$ & $1.5-7.0$ \\
\hline Attentional focusing & $3.7(1.1)$ & $1.0-6.8$ \\
\hline Attentional shifting & $4.6(0.8)$ & $1.8-6.9$ \\
\hline Cuddliness & $4.1(1.1)$ & $1.5-7.0$ \\
\hline Inhibitory control & $3.1(0.9)$ & $1.0-6.1$ \\
\hline Low-intensity pleasure & $4.7(0.8)$ & $1.8-6.7$ \\
\hline Activity level & $4.9(0.8)$ & $2.1-7.0$ \\
\hline High-intensity pleasure & $4.2(1.6)$ & $1.0-7.0$ \\
\hline Impulsivity & $4.3(1.3)$ & $1.0-7.0$ \\
\hline Positive anticipation & $3.9(0.9)$ & $1.0-5.0$ \\
\hline Sociability & $4.7(1.9)$ & $1.0-7.0$ \\
\hline Expressive language scores (z-score) & $-0.01(1.02)$ & $-3.00-2.88$ \\
\hline Receptive language scores (z-score) & $-0.02(0.98)$ & $-3.00-3.00$ \\
\hline
\end{tabular}

Note. ECBQ Early Child Behavior Questionnaire

associations were nonsignificant. Even after controlling for all covariates (Model 3), two subscales remained significantly associated with receptive language scores: the motor activation subscale was negatively associated with receptive language scores, and the perceptual sensitivity subscale was positively associated with receptive language scores.

\section{Discussion}

This study investigated whether child temperament at 18 months of age, measured with the ECBQ, predicted expressive and receptive language skills at 40 months. The motor activation and perceptual sensitivity temperament subscales at 18 months predicted both expressive and receptive language skills at 40 months.

\section{Motor activation subscale and language skills}

We found that higher scores on the motor activation subscale predicted lower scores for both expressive and receptive language skills. This could be explained by the subscale being defined as fidgeting (Table 1), as children who fidget repetitively at 18 months are assumed to generally have limited language skills during early childhood. This is consistent with the observation provided by Garello et al. [45], who demonstrated that high scores on the motor activation subscale at 24-30 months were 
Table 3 Associations of subscales of the Early Child Behavior Questionnaire and expressive language scores at 40 months: regression coefficients in z-score (change in SD), 95\% confidence intervals and $p$-values

\begin{tabular}{|c|c|c|c|c|c|c|}
\hline & \multicolumn{2}{|l|}{ Model 1} & \multicolumn{2}{|l|}{ Model 2} & \multicolumn{2}{|l|}{ Model 3} \\
\hline & Coefficient $[95 \% \mathrm{Cl}]$ & $p$ & Coefficient $[95 \% \mathrm{Cl}]$ & $p$ & Coefficient $[95 \% \mathrm{Cl}]$ & $p$ \\
\hline Motor activation & $-\mathbf{0 . 1 9 2}[-0.274,-0.109]$ & $<.001$ & $-0.234[-0.331,-0.137]$ & $<.001$ & $-0.211[-0.305,-0.117]$ & $<.001$ \\
\hline Perceptual sensitivity & $\mathbf{0 . 1 4 4}[0.079,0.209]$ & $<.001$ & $\mathbf{0 . 1 5 7}[0.079,0.236]$ & $<.001$ & $\mathbf{0 . 1 3 7}[0.061,0.213]$ & $<.001$ \\
\hline Inhibitory control & $\mathbf{0 . 1 9 0}[0.115,0.265]$ & $<.001$ & $0.110[0.024,0.195]$ & .01 & $0.091[0.009,0.173]$ & .029 \\
\hline Soothability & $\mathbf{0 . 1 1 9}[0.048,0.189]$ & .001 & $0.028[-0.055,0.110]$ & .51 & $0.024[-0.054,0.103]$ & .55 \\
\hline Attentional shifting & $\mathbf{0 . 2 3 2}[0.146,0.317]$ & $<.001$ & $0.095[-0.005,0.194]$ & .06 & $0.087[-0.008,0.182]$ & .07 \\
\hline Low-intensity pleasure & $\mathbf{0 . 2 0 6}[0.116,0.296]$ & $<.001$ & $0.105[0.008,0.202]$ & .034 & $0.066[-0.026,0.159]$ & .16 \\
\hline
\end{tabular}

Note. Model 1 = Univariate; Model 2 =Adjusted for other subscales of the Early Child Behavior Questionnaire Subscales (ECBQ); Model $3=$ Model 2 with further adjustment for child sex, birth weight, gestational age at birth, birth order, age of the mother, years of maternal education, annual household income, maternal history of mood/anxiety disorders. Cl Confidence intervals. Bold types represent $p<.0013$. The regression coefficients shown in Table 3 indicate the amount of predicted change in the $z$-score of expressive language skills per one unit of change in each of the ECBQ subscales. The marginal magnitude of difference was calculated as follows: a child scoring 7 (maximum) in motor activation, for example, was predicted to score $-0.211 \times 6$ (i.e., 7 minus 1 point in the subscale) $=-1.266$ points, corresponding to -1.266 SD higher lower in the expressive language skill score at 40 months compared with a child scoring 1 (minimum)

Table 4 Associations of subscales of the Early Child Behavior Questionnaire and receptive language scores at 40 months: regression coefficients in z-score (change in SD), 95\% confidence intervals and $p$-values

\begin{tabular}{|c|c|c|c|c|c|c|}
\hline & \multicolumn{2}{|l|}{ Model 1} & \multicolumn{2}{|l|}{ Model 2} & \multicolumn{2}{|l|}{ Model 3} \\
\hline & Coefficient $[95 \% \mathrm{Cl}]$ & $p$ & Coefficient $[95 \% \mathrm{Cl}]$ & $p$ & Coefficient $[95 \% \mathrm{Cl}]$ & $p$ \\
\hline Motor activation & $\mathbf{- 0 . 1 7 4}[-0.254,-0.095]$ & $<.001$ & $-\mathbf{0 . 2 4 5}[-0.339,-0.150]$ & $<.001$ & $-\mathbf{0 . 2 2 5}[-0.317,-0.133]$ & $<.001$ \\
\hline Perceptual sensitivity & $\mathbf{0 . 1 3 2}[0.069,0.195]$ & $<.001$ & $\mathbf{0 . 1 6 9}[0.092,0.245]$ & $<.001$ & $\mathbf{0 . 1 5 0}[0.076,0.225]$ & $<.001$ \\
\hline Attentional shifting & $\mathbf{0 . 1 7 8}[0.095,0.262]$ & $<.001$ & $0.071[-0.026,0.168]$ & .15 & $0.063[-0.030,0.156]$ & .18 \\
\hline Inhibitory control & $\mathbf{0 . 1 4 5}[0.072,0.218]$ & $<.001$ & $0.082[-0.001,0.165]$ & .05 & $0.066[-0.015,0.146]$ & .11 \\
\hline
\end{tabular}

Note. Model 1 = Univariate; Model 2 =Adjusted for other subscales of the Early Child Behavior Questionnaire Subscales (ECBQ); Model $3=$ Model 2 with further adjustment for child sex, birth weight, gestational age at birth, birth order, age of the mother, years of maternal education, annual household income, maternal history of mood/anxiety disorders. Cl Confidence intervals. Bold types represent $p<.0013$. The regression coefficients shown in Table 4 indicate the amount of predicted change in the $z$-score of receptive language skills per one unit of change in each of the ECBQ subscales. Again, this implies that a child scoring 7 (maximum) in motor activation, for example, was predicted to score $-0.225 \times 6$ ( 7 minus 1 point in the subscale) $=-1.350$ points, corresponding to -1.350 SD higher lower in the receptive language skills score at 40 months compared with a child scoring 1 (minimum)

associated with lower expressive language skills when measured simultaneously. In addition, Berger et al. [46] found that poor motor adjustment during early childhood, a representation of poor ability to sit still, required early attentional skills for performing tasks at hand. This makes it difficult for children to learn language skills [47]. We have expanded the work done by Garello et al. [45] by providing evidence that fidgeting behavior at 18 months, perhaps due to the cost of attentional resources, predicts poorer language skills at 40 months.

\section{Perceptual sensitivity subscale and language skills}

Higher scores on the perceptual sensitivity subscale predicted higher scores for both expressive and receptive language skills at 40 months. Perceptual sensitivity is a construct that reflects the ability to detect slight, lowintensity stimuli in the external environment (Table 1). Specifically, such stimuli in the external environment cover several modality areas including auditory, visual, and tactile stimuli, although most of the questions categorized in this ECBQ subscale are related to auditory stimuli [25]. Notably, it is suggested that music, a broad set of patterned auditory stimuli, enhances speech perception in infancy [48], which predicts expressive and receptive language skills in later years [49]. It is likely that children exhibiting better performance in auditory perception achieve advanced language skills in later years. Furthermore, studies have consistently suggested that perceptual sensitivity is a reflection of early attentional skills $[25,50]$, which drive children to selectively attend to subtle stimuli and respond to them with their voices [51]. To this end, we presume that children with higher scores on the perceptual sensitivity subscale can better attend to and handle external stimuli, particularly auditory stimuli, which is reflected in their higher scores in language skills during early childhood.

\section{Relevance of early attention to the findings}

Higher scores in motor activation and lower scores in perceptual sensitivity both accompanied problems in 
early attentional skills. The original studies indicated that attentional skills might emerge as early as the first year of life [21] and serve as a foundation of behavioral control over reactivity [51]. As such, higher scores in motor activation subscale involve difficulties in sitting still [46], and lower scores in perceptual sensitivity subscale involve poor response to external stimuli, including human voices [51]. These examples highlight the significant role of early attentional skills in language learning [30,52].

\section{Subscales that have no association with language skills}

Unlike the relevance of early attentional skills to the two subscales (motor activation and perceptual sensitivity) that predicted language skills at 40 months, other attention-related subscales (attentional focusing and attentional shifting subscales) did not similarly predict language skills. To illustrate, the two subscales were marginally or significantly associated with both expressive and receptive language skills at 40 months only in Model 1, although the associations became insignificant after adjusting for the other subscales (Supplementary Tables 3 and 4). Our findings are inconsistent with several studies reporting the relevance of two attentional skills (attentional focusing and shifting) to language development [53], although the methodologies employed were different. More importantly, studies by the developers of ECBQ have shown that the attentional focusing and shifting subscales are categorized as "executive attention" and that these two skills emerge as an early stage of development in self-regulation prominent in later years (approximately 4 years) [25, 27, 29]. Executive attention was defined as "mechanisms for monitoring and resolving conflict among thoughts, feelings, and responses" [54] and was suggested to form the basis of executive function [55]. Thus, the attentional focusing and shifting subscales may not be interpreted in the same way as the motor activation and perceptual sensitivity subscales representing early attention emerging during the first year of life. In other words, the association of early temperament and later language skills is age-specific; while the attentional focusing and shifting subscales at 18 months are not predictors for language skills at 40 months, the motor activation and perceptual sensitivity subscales at 18 months specifically predict level of language skills at 40 months.

\section{Other factors that may explain our findings}

External factors should also be considered when interpreting our results. That is, language skills during early childhood have been reported to be influenced by child factors such as sex [8], birth weight [9], gestational age at birth [10], and birth order [11]. Maternal factors, such as age [12], years of education [13], and history of mood or anxiety disorders $[14,15]$ have also been found to influence language skills during early childhood. Even after controlling for these factors, the associations remained significant (see Supplementary Tables 3 and 4). This suggests that the associations between specific child temperament and language skills are generally independent of known external factors, although we did not control for all external factors (e.g., attachment [56] and parenting [57]).

\section{Clinical implications and future directions}

Lower scores on the motor activation subscale and higher scores on the perceptual sensitivity subscale predict advantageous courses in language development in expressive and receptive skills. These findings are beneficial for child health professionals to predict sufficient language development during early childhood and to identify children at potential risk of insufficient language development. This is because early child temperament can be easily measured without any expertise and with a range of available tools, including the full and short versions of the ECBQ [58].

Future studies are expected to elucidate potential biological mechanisms underlying the association between child temperament and language skills. Genetic factors may be one account for the associations we observed. Although genes that account for the association between temperament and language skills have not yet been reported, expressive and receptive language skills are influenced by genetic factors [59]; child temperament is also genetically determined [22].

\section{Limitations and strengths}

The present study has several limitations that should be considered when interpreting the results. First, approximately $28 \%$ of participants were excluded from the analysis, although comparisons between the analyzed and excluded participants did not reveal significant differences except for mothers' age and household income. Second, we conducted conservative hypothesis tests. While one may set the significance level more loosely using, e.g., the false discovery rate, we did not do this because of our explorative study design which did not propose any hypothesis in advance. Third, although we considered a range of potential confounders or covariates, other variables that could have been controlled for, for example, attachment [56], parenting [57], family history of language delay [13], child-directed speech [60], and home literacy environment [61] were not measured, as their roles were not pivotal (e.g., [32, 62]). Fourth, the ECBQ is a parent-reported questionnaire, which may have led to information bias. For example, parents whose children showed language delays might have given lower 
scores on the ECBQ. To minimize this bias, we confirmed the inputs from the caregivers by conducting interviews with them. Finally, child temperament measures using the ECBQ were assessed at one time point.

Despite these limitations, the strengths of this study include its longitudinal design with a larger sample size compared to existing studies. Moreover, it focused on each subscale of the ECBQ while controlling for other subscales and covariates that have been found to relate to temperament and language.

\section{Conclusions}

The present study found that specific temperamental subscales-motor activation and perceptual sensitivity-at of age. These results remained significant even when controlling for other potential confounding factors, suggesting the relevance of specific temperament in language development and that these temperament subscales are useful for predicting language development in children. Taken together, our findings show that temperament, especially in areas of motor activation and perceptual sensitivity, can aid clinicians in evaluating the potential risk of insufficient language development later in children and provide opportunities for early interventions.

\section{Abbreviations}

ECBQ: Early Childhood Behavior Questionnaire; HBC Study: Hamamatsu Birth Cohort for Mothers and Children; MSEL: Mullen Scales of Early Learning.

\section{Supplementary Information}

The online version contains supplementary material available at https://doi. of the children and parents excluded in the analysis. Supplementary Table 2. The Pearson correlation matrix of the 18 subscales of the Early Child Behavior Questionnaire. Supplementary Table 3. Associations of 18 subscales of the Early Child Behavior Questionnaire and expressive confidence intervals and $p$-values. Supplementary Table 4. Associations of 18 subscales of the Early Child Behavior Questionnaire and receptive language scores 40 months: regression coefficients in z-score, 95\% confidence intervals and $p$-values. 18 months of age predicted language skills at 40 months org/10.1186/s12887-022-03116-5.

Additional file 1: Supplementary Table 1. Demographic characteristics language scores at 40 months: regression coefficients in z-score, 95\%

Iwata, E Kawai, M Kawai, YK Kuroda, K Matsumoto, H Matsuzaki, N Mori, T Mori, K Nakaizumi, K Nakamura, AA Pillai, Y Seno, C Shimmura, S Suda, G Sugihara, T Sugiyama, K Suzuki, K Yamada, S Yamamoto, Y Yoshihara, Y Endoh, K Hirano, and T Suzuki. We would like to thank Editage for English language editing.

\section{Authors' contributions}

$\mathrm{YI}-\mathrm{O}$ and $\mathrm{KJT}$ conceptualized the study, performed the formal analysis, and drafted and edited the manuscript. TN, AO, TH, TI, CN, YA, HS, and RN participated in the investigation, data curation, review, and editing of the paper. AN, MSR, NT, and YN conceptualized the study and edited the manuscript. All authors read and approved the final manuscript.

\section{Funding}

This study was supported by Grants-in-Aid for Scientific Research (B) $[19 \mathrm{H} 03582, \mathrm{KJT}]$ and was conducted under the framework of the Collaborative Research Network for Asian Children with Developmental Disorders ("CRNACDD"), Osaka University United Graduate School of Child Development.

\section{Availability of data and materials}

The analysis code compiled using Stata software is available from the corresponding author. Data sharing with readers is not available because of the requirements of the local Ethical Review Board.

\section{Declarations}

\section{Ethics approval and consent to participate}

The study was conducted in accordance with the guidelines proposed in the World Medical Association Declaration of Helsinki and was approved by the Ethical Committee of Hamamatsu University School of Medicine (No. 20-82, 21-114, 22-29, 24-67, 24-237, 25-143, 25-283, E14-062, 17-037, 17-037-3, 20-233). Written informed consent was obtained from all caregivers for their own participation and that of their infants.

\section{Consent for publication}

Not applicable.

\section{Competing interests}

The authors declare that they have no competing interests.

\section{Author details}

${ }^{1}$ Department of Child Development, United Graduate School of Child Development, Osaka University, Kanazawa University, Hamamatsu University School of Medicine, Chiba University, and University of Fukui, Handayama 1 Higashiku, Hamamatsu 4313192, Japan. ${ }^{2}$ Research Center for Child Mental Development, Hamamatsu University School of Medicine, Handayama 1 Higashiku, Hamamatsu 4313192, Japan. ${ }^{3}$ Graduate School of Humanities and Social Sciences, Nagoya City University, Mizuho-cho Yamanohata 1, Mizuho-ku, Nagoya 4678501, Japan. ${ }^{4}$ Department of Psychology, Queens College, City University of New York, 65-30 Kissena Blvd, Flushing, New York, NY 11367 , USA. ${ }^{5}$ Department of Psychiatry, Icahn School of Medicine at Mount Sinai, 1 Gustave L. Levy Place, New York, NY 10029, USA.

Received: 15 June 2021 Accepted: 10 January 2022

Published online: 21 January 2022

\section{Acknowledgments}

The authors thank Dr. Tetsuo Kato of the Kato Maternity Clinic, as well as Drs. K Sugihara, M Sugimura, K Takeuchi, K Suzuki, Y Murakami, Y Kohmura, Y Miyabe, K Hirai, Y Nakamura, R Koizumi, H Murakami, Y Kobayashi-Kohmura, K Muramatsu-Kato, Prof. H Itoh, Prof. N Kanayama, and all attending obstetricians of Hamamatsu University School of Medicine, for their full support with participant enrolment in the study. The authors also thank the chief midwife, Ms. Kiyomi Hinoki, and all midwives and staff at the maternity clinic of Hamamatsu University School of Medicine, for their support with participant enrolment. The HBC Study team includes E Higashimoto, N Kodera, A Nakamura, Y Nishimura, Y Kugizaki, Y Suzuki, R Takabayashi, M Honda, H Muraki, M Narumiya, E Sato, Drs. D Choi, T Horikoshi, M Tsujii, K Wakusawa, Y Kameno, D Kurita, H Kuwabara, K Takebayashi, M Yokokura, TWakuda, R Asano, T Ismail, K Iwata, Y

\section{References}

1. Rogers CR, Nulty KL, Betancourt MA, DeThorne LS. Causal effects on child language development: a review of studies in communication sciences and disorders. J Commun Disord. 2015:57:3-15.

2. Sakai KL. Language acquisition and brain development. Science. 2005;310(5749):815-9.

3. Conti-Ramsden G, Durkin K. Language development and assessment in the preschool period. Neuropsychol Rev. 2012;22(4):384-401.

4. Norbury CF, Vamvakas G, Gooch D, Baird G, Charman T, Simonoff E, et al. Language growth in children with heterogeneous language disorders: a population study. J Child Psychol Psychiatry. 2017;58(10):1092-105. 
5. Young AR, Beitchman JH, Johnson C, Douglas L, Atkinson L, Escobar $M$, et al. Young adult academic outcomes in a longitudinal sample of early identified language impaired and control children. J Child Psychol Psychiatry. 2002;43(5):635-45.

6. Bornstein MH, Hahn CS, Putnick DL, Pearson RM. Stability of core language skill from infancy to adolescence in typical and atypical development. Sci Adv. 2018;4(11):eaat7422.

7. Ukoumunne OC, Wake M, Carlin J, Bavin EL, Lum J, Skeat J, et al. Profiles of language development in pre-school children: a longitudinal latent class analysis of data from the early language in Victoria study. Child Care Health Dev. 2012;38(3):341-9.

8. Law J, Clegg J, Rush R, Roulstone S, Peters TJ. Association of proximal elements of social disadvantage with children's language development at 2 years: an analysis of data from the children in focus (CiF) sample from the ALSPAC birth cohort. Int I Lang Commun Disord. 2019;54(3):362-76.

9. Barre N, Morgan A, Doyle LW, Anderson PJ. Language abilities in children who were very preterm and/or very low birth weight: a meta-analysis. J Pediatr. 2011;158(5):766-74 e1.

10. Putnick DL, Bornstein MH, Eryigit-Madzwamuse S, Wolke D. Long-Term Stability of Language Performance in Very Preterm, Moderate-Late Preterm, and Term Children. J Pediatr. 2017;181:74-9 e3.

11. Hoff E. How social contexts support and shape language development. Dev Rev. 2006;26:55-88.

12. Sutcliffe AG, Barnes J, Belsky J, Gardiner J, Melhuish E. The health and development of children born to older mothers in the United Kingdom: observational study using longitudinal cohort data. BMJ. 2012;345:e5116.

13. Reilly S, Wake M, Ukoumunne OC, Bavin E, Prior M, Cini E, et al. Predicting language outcomes at 4 years of age: findings from early language in Victoria study. Pediatrics. 2010;126(6):e1530-7.

14. Aoyagi SS, Takei N, Nishimura T, Nomura Y, Tsuchiya KJ. Association of late-onset postpartum depression of mothers with expressive language development during infancy and early childhood: the HBC study. PeerJ. 2019;7:e6566.

15. Reck C, Van Den Bergh B, Tietz A, Müller M, Ropeter A, Zipser B, et al. Maternal avoidance, anxiety cognitions and interactive behaviour predicts infant development at 12 months in the context of anxiety disorders in the postpartum period. Infant Behav Dev. 2018;50:1 16-31.

16. Dixon WE, Smith PH. Links between early temperament and language acquisition. Merrill-Palmer Q. 2000;46:417-40.

17. Pérez-Pereira M, Fernández P, Resches M, Gómez-Taibo ML. Does temperament influence language development? Evidence from preterm and full-term children. Infant Behav Dev. 2016:42:11-21.

18. Smith Watts AK, Patel D, Corley RP, Friedman NP, Hewitt JK, Robinson JL, et al. Testing alternative hypotheses regarding the association between behavioral inhibition and language development in toddlerhood. Child Dev. 2014;85(4):1569-85.

19. Kristal J. The temperament perspective: working with children's behavior styles. New York: Brookes Publishing Co; 2005.

20. Goldsmith HH. Studying temperament via construction of the toddler behavior assessment questionnaire. Child Dev. 1996;67(1):218-35.

21. Rothbart MK. Becoming who we are: temperament and personality in development. New York: Guilford Press; 2011.

22. Posner MI, Rothbart MK. Temperament and brain networks of attention. Philos Trans R Soc Lond Ser B Biol Sci. 2018;373(1744):20170254.

23. Conture EG, Kelly EM, Walden TA. Temperament, speech and language: an overview. J Commun Disord. 2013;46(2):125-42.

24. Gartstein MA, Rothbart MK. Studying infant temperament via the revised infant behavior questionnaire. Infant Behav Dev. 2003;26:64-86.

25. Putnam SP, Gartstein MA, Rothbart MK. Measurement of fine-grained aspects of toddler temperament: the early childhood behavior questionnaire. Infant Behav Dev. 2006;29(3):386-401.

26. Putnam SP, Rothbart MK, Gartstein MA. Homotypic and heterotypic continuity of fine-grained temperament during infancy, toddlerhood, and early childhood. Infant Child Dev. 2008;17:387-405

27. Posner MI, Rothbart MK, Sheese BE, Voelker P. Developing attention: behavioral and brain mechanisms. Adv Neurosci. 2014:2014:405094

28. Petersen IT, Bates JE, Staples AD. The role of language ability and selfregulation in the development of inattentive-hyperactive behavior problems. Dev Psychopathol. 2015;27(1):221-37.

29. Posner MI, Rothbart MK, Voelker P. Developing brain networks of attention. Curr Opin Pediatr. 2016;28(6):720-4
30. Vouloumanos A, Curtin S. Foundational tuning: how infants' attention to speech predicts language development. Cogn Sci. 2014;38(8):1675-86.

31. Davison L, Warwick H, Campbell K, Gartstein M. Infant temperament affects toddler language development. J Educ E-learn Res. 2019;6:122-8.

32. Spinelli M, Fasolo M, Shah PE, Genovese G, Aureli T. The influence of early temperament on language development: the moderating role of maternal input. Front Psychol. 2018;9:1527.

33. Usai MC, Viterbori P, Gandolfi E, Zanobini M. The relationship between temperamental dimensions and inhibitory control in early childhood: implications for language acquisition. Infant Behav Dev. 2020;61:101495.

34. Hilton M, Westermann G. The effect of shyness on children's formation and retention of novel word-object mappings. J Child Lang. 2017:44(6):1394-412.

35. Kucker SC, Zimmerman C, Chmielewski M. Taking parent personality and child temperament into account in child language development. Br J Dev Psychol. 2021;39(4):540-65.

36. Laake LM, Bridgett DJ. Happy babies, chatty toddlers: infant positive affect facilitates early expressive, but not receptive language. Infant Behav Dev. 2014;37(1):29-32.

37. Takagai S, Tsuchiya KJ, Itoh H, Kanayama N, Mori N, Takei N, et al. Cohort profile: Hamamatsu birth cohort for mothers and children (HBC study). Int J Epidemiol. 2016;45(2):333-42.

38. Tsuchiya KJ, Matsumoto K, Suda S, Miyachi H, Itoh N, Kanayama K, et al. Searching for very early precursors of autism spectrum disorders: the Hamamatsu birth cohort for mothers and children (HBC). J Dev Orig Health Dis. 2010;1 (3):158-73.

39. Sukigara M, Nakagawa A, Mizuno R. Development of a Japanese version of the early childhood behavior questionnaire (ECBQ) using cross-sectional and longitudinal data. SAGE Open. 2015;5:1-12.

40. Mullen EM. Mullen scales of early learning. AGS ed. Minnesota: Circle Pines; 1995.

41. Almekaini LA, Zoubeidi T, Albustanji Y. Screening for speech-language development in Emirati toddlers. J Psychol Cognition. 2017;2(1):26-31.

42. Nishimura T, Takei N, Tsuchiya KJ, Asano R, Mori N. Identification of neurodevelopmental trajectories in infancy and of risk factors affecting deviant development: a longitudinal birth cohort study. Int J Epidemiol. 2016;45(2):543-53.

43. Groenwold RH, Klungel OH, Grobbee DE, Hoes AW. Selection of confounding variables should not be based on observed associations with exposure. Eur J Epidemiol. 2011:26(8):589-93.

44. Rubin M. Do $p$ values lose their meaning in exploratory analyses? It depends how you define the familywise error rate. Rev Gen Psychol. 2017:21:269-75.

45. Garello V, Viterbori P, Usai MC. Temperamental profiles and language development: a replication and an extension. Infant Behav Dev. 2012;35(1):71-82.

46. Berger SE, Harbourne RT, Guallpa Lliguichuzhca CL. Sit still and pay attention! Trunk movement and attentional resources in infants with typical and delayed development. Phys Occup Ther Pediatr. 2019;39(1):48-59.

47. Norbury CF. Sources of variation in developmental language disorders: evidence from eye-tracking studies of sentence production. Philos Trans R Soc Lond Ser B Biol Sci. 2014;369(1634):20120393.

48. Zhao TC, Kuhl PK. Musical intervention enhances infants' neural processing of temporal structure in music and speech. Proc Natl Acad Sci U S A. 2016;113:5212-7.

49. Zhao TC, Boorom O, Kuhl PK, Gordon R. Infants' neural speech discrimination predicts individual differences in grammar ability at 6 years of age and their risk of developing speech-language disorders. Dev Cogn Neurosci. 2021;48:100949.

50. Petersen SE, Posner MI. The attention system of the human brain: 20 years after. Annu Rev Neurosci. 2012;35:73-89.

51. Gartstein MA, Crawford J, Robertson CD. Early markers of language and attention: mutual contributions and the impact of parent-infant interactions. Child Psychiatry Hum Dev. 2008;39(1):9-26.

52. Nakagawa A, Sukigara M. Individual differences in disengagement of fixation and temperament: Iongitudinal research on toddlers. Infant Behav Dev. 2013;36(4):728-35.

53. Mundy P, Block J, Delgado C, Pomares Y, Van Hecke AV, Parlade MV. Individual differences and the development of joint attention in infancy. Child Dev. 2007;78:938-54. 
54. Posner Ml, Rothbart MK. Research on attention networks as a model for the integration of psychological science. Annu Rev Psychol. 2007;58:1-23.

55. Conejero A, Rueda MR. Early development of executive attention. J Child Adolesc Behav. 2017;5:341.

56. Groh AM, Narayan AJ, Bakermans-Kranenburg MJ, Roisman GI, Vaughn BE, Fearon RMP, et al. Attachment and temperament in the early life course: a Meta-analytic review. Child Dev. 2017;88(3):770-95.

57. Garcia D, Rodriquez GM, Hill RM, Lorenzo NE, Bagner DM. Infant language production and parenting skills: a randomized controlled trial. Behav Ther. 2019;50(3):544-57.

58. Putnam SP, Jacobs JF, Gartstein MA, Rothbart MK. Development and assessment of short and very short forms of the Early Childhood Behavior Questionnaire. Baltimore: Poster presented at the annual International Conference on Infant Studies; 2010.

59. Hayiou-Thomas ME, Dale PS, Plomin R. The etiology of variation in language skills changes with development: a longitudinal twin study of language from 2 to 12 years. Dev Sci. 2012;15(2):233-49.

60. Golinkoff RM, Can DD, Soderstrom M, Hirsh-Pasek K. (Baby)talk to me: The social context of infant-directed speech and its effects on early language acquisition. Curr Dir Psychol Sci. 2015:24:339-44.

61. Roberts J, Jurgens J, Burchinal M. The role of home literacy practices in preschool children's language and emergent literacy skills. J Speech Lang Hear Res. 2005;48(2):345-59.

62. Kidd E, Donnelly S. Individual differences in first language acquisition. Annu Rev Linguist. 2020;6:319-40.

\section{Publisher's Note}

Springer Nature remains neutral with regard to jurisdictional claims in published maps and institutional affiliations.

Ready to submit your research? Choose BMC and benefit from:

- fast, convenient online submission

- thorough peer review by experienced researchers in your field

- rapid publication on acceptance

- support for research data, including large and complex data types

- gold Open Access which fosters wider collaboration and increased citations

- maximum visibility for your research: over 100M website views per year

At BMC, research is always in progress.

Learn more biomedcentral.com/submissions 